\title{
The unique solution for periodic differential equations with upper and lower solutions in reverse order
}

\section{Aijun Yang ${ }^{*}$, Helin Wang ${ }^{*}$ and Dingjiang Wang}

"Correspondence:

yangaij2004@163.com; wh1982032@163.com

College of Science, Zhejiang

University of Technology,

Hangzhou, Zhejiang 310023,

P.R. China

\section{Abstract}

In this paper, we obtain the unique solution for the following periodic problem

$$
\begin{aligned}
& x^{\prime \prime}(t)=g\left(t, x(t), x^{\prime}(t)\right), \quad t \in(a, b), \\
& x(a)=x(b), \quad x^{\prime}(a)=x^{\prime}(b),
\end{aligned}
$$

where $g:[a, b] \times \mathbb{R}^{2} \rightarrow \mathbb{R}$ is a continuous function, by constructing an auxiliary system with bounded solutions. The upper and lower solution method and an anti-maximum principle are employed to establish the monotone iterative sequences and obtain the extremal solutions for the auxiliary system.

MSC: $34 \mathrm{~B} 15 ; 34 \mathrm{C} 25$

Keywords: reversed order upper and lower solutions; monotone iterative method; anti-maximum principle; unique solution

\section{Introduction}

This paper is concerned with the unique solution to the following periodic problem:

$$
\left\{\begin{array}{l}
x^{\prime \prime}(t)=g\left(t, x(t), x^{\prime}(t)\right), \quad t \in(a, b), \\
x(a)=x(b), \quad x^{\prime}(a)=x^{\prime}(b),
\end{array}\right.
$$

where $g:[a, b] \times \mathbb{R}^{2} \rightarrow \mathbb{R}$ is a continuous function.

It is well known that the upper and lower solution method together with the iterative technique is a powerful tool for proving the existence results for boundary value problems (see [1-12] and the references therein). Recently, the case when the upper solution and the lower solution are in the reversed order has received some attention (see [2-9] and the references therein). The monotone approximation method can be used in the case the lower and upper solutions are in the reversed order $\beta \leq \alpha$. This method works for any boundary value problem such that a uniform anti-maximum principle holds. This is the case for the Neumann and periodic problems. 
For example, Cabada et al. in [3] used iteration schemes based on problems like

$$
\left\{\begin{array}{l}
\alpha_{n}^{\prime \prime}-2 k\left|\alpha_{n}^{\prime}-\alpha_{n-1}^{\prime}\right|+\lambda \alpha_{n}=f\left(t, \alpha_{n-1}, \alpha_{n-1}^{\prime}\right)+\lambda \alpha_{n-1}, \\
\alpha_{n}^{\prime}(a)=\alpha^{\prime}(b)=0
\end{array}\right.
$$

discussed a Neumann boundary value problem

$$
\left\{\begin{array}{l}
u^{\prime \prime}(t)=f\left(t, u(t), u^{\prime}(t)\right), \quad t \in[a, b], \\
x^{\prime}(a)=x^{\prime}(b)=0 .
\end{array}\right.
$$

In [4], Torres and Zhang considered a kind of $2 \pi$-periodic boundary value problem. The strategy of this paper was to exploit an anti-maximum principle for the linear equation in order to construct a monotone approximation scheme converging to the solution.

In [8], Zuo et al. further developed the monotone method and invested the $T$-periodic solution of

$$
y^{\prime \prime}(t)=f(t, y(t), y(w(t))), \quad t \in \mathbb{R} .
$$

They used the monotone iterative technique with upper and lower solution in reversed order to define two sequences that converge uniformly to extremal solution of (1.2). However, they did not construct the explicit expression of the monotone iterative sequences.

We have investigated the periodic problems (1.1) in [13] by introducing the following auxiliary periodic system:

$$
\left\{\begin{array}{l}
u^{\prime \prime}(t)=f\left(t, u(t), u^{\prime}(t), v(t)\right), \\
v^{\prime \prime}(t)=f\left(t, v(t), v^{\prime}(t), u(t)\right), \\
u(a)=u(b), \quad u^{\prime}(a)=u^{\prime}(b), \\
v(a)=v(b), \quad v^{\prime}(a)=v^{\prime}(b),
\end{array}\right.
$$

where $f:[a, b] \times \mathbb{R}^{3} \rightarrow \mathbb{R}$ is a $L^{1}$-Carathéodory function, and $f(t, u, w, u) \equiv g(t, u, w)$.

Motivated by the above works, we are mainly concerned with the periodic problem (1.1). One might have noticed that computing the iterative sequences $\left\{\alpha_{n}\right\}$ and $\left\{\beta_{n}\right\}$ can be a difficult work in those existed papers. In this paper, by adopting an auxiliary periodic system and an anti-maximum principle which are different from that in the reference [13], we also obtain the unique solution for the problem (1.1).

Here, we introduce the following auxiliary periodic system:

$$
\left\{\begin{array}{l}
u^{\prime \prime}(t)+K u(t)=f\left(t, u(t), u^{\prime}(t), v(t)\right)+K v(t), \quad t \in(a, b), \\
v^{\prime \prime}(t)+K v(t)=f\left(t, v(t), v^{\prime}(t), u(t)\right)+K u(t), \quad t \in(a, b), \\
u(a)=u(b), \quad u^{\prime}(a)=u^{\prime}(b), \\
v(a)=v(b), \quad v^{\prime}(a)=v^{\prime}(b),
\end{array}\right.
$$

where $f:[a, b] \times \mathbb{R}^{3} \rightarrow \mathbb{R}$ is a continuous function and $f(t, u, w, u)=g(t, u, w)$, the constant $K$ is defined in Section 3. 
The organization of this paper is as follows. We shall introduce some useful lemmas in Section 2. The main results and their proof about auxiliary periodic (1.3) are given in Section 3. Section 4 gives the unique solution of (1.1) and an example is introduced.

\section{Related lemmas}

For the convenience of the reader, we give some lemmas which will be used in the next section. Firstly, recall the continuation theorem of Mawhin.

Lemma 2.1 [12] Let $X$ and $Y$ be two Banach spaces with norms $\|\cdot\|_{X}$ and $\|\cdot\|_{Y}$, respectively, and $\Omega \subset X$ an open and bounded set. Suppose $L: X \cap \operatorname{dom} L \rightarrow Y$ is a Fredholm operator of index zero and $N_{\lambda}: \bar{\Omega} \rightarrow Y, \lambda \in[0,1]$ is L-compact. In addition, if

(A1) $L x \neq \lambda N x$ for $\lambda \in(0,1), x \in(\operatorname{dom} L \backslash \operatorname{ker} L) \cap \partial \Omega$;

(A2) $N x \notin \operatorname{Im} L$ for $x \in \operatorname{ker} L \cap \partial \Omega$;

(A3) $\operatorname{deg}\left\{\left.J Q N\right|_{\bar{\Omega} \cap \operatorname{ker} L}, \Omega \cap \operatorname{ker} L, 0\right\} \neq 0$, where $Q: Y \rightarrow Y$ is a projection such that $\operatorname{Im} L=\operatorname{ker} Q$ and $J: \operatorname{Im} Q \rightarrow \operatorname{ker} L$ is a homeomorphism.

Then the abstract equation $L x=N x$ has at least one solution in $\bar{\Omega}$.

Secondly, to prove the validity of the monotone iterative technique, we present the antimaximum comparison principle as follows.

Lemma $2.2[1]$ Let $p \in \mathbb{R}, q>0, \sigma \in L^{1}(a, b)$ and $A \in \mathbb{R}$. Suppose $u$ is a solution of

$$
\left\{\begin{array}{l}
x^{\prime \prime}(t)+2 p\left|x^{\prime}(t)\right|+q x(t)=\sigma(t), \quad t \in(a, b), \\
x(a)-x(b)=0, \quad x^{\prime}(a)-x^{\prime}(b)=A
\end{array}\right.
$$

for some $A \geq 0$ and $\sigma \geq 0$. Then $u \geq 0$ provided that $p \geq 0$ and $\theta(q, p) \geq \frac{b-a}{2}$, where

$$
\theta(q, p)= \begin{cases}\frac{1}{\sqrt{p^{2}-q}} \operatorname{arctanh} \frac{\sqrt{p^{2}-q}}{p}, & 0<q<p^{2}, \\ \frac{1}{p}, & 0<q=p^{2}, \\ \frac{1}{\sqrt{q-p^{2}}}\left(\frac{\pi}{2}-\arctan \frac{p}{\sqrt{q-p^{2}}}\right), & 0 \leq p^{2}<q .\end{cases}
$$

Finally, recall some classical integral inequalities.

Lemma $2.3[14]$ If $u \in C^{1}[a, b]$ is such that $\bar{u}=0$, where $\bar{u}=\frac{1}{b-a} \int_{a}^{b} u(s) d s$. Then

$$
\|u\|_{2} \leq \frac{b-a}{\pi}\left\|u^{\prime}\right\|_{2}, \quad\|u\|_{\infty} \leq \sqrt{b-a}\left\|u^{\prime}\right\|_{2}
$$

\section{Extremal solution for (1.3)}

Functions $\alpha, \beta \in C^{2}[a, b]$ are said to be a pair of lower and upper solutions to (1.3) if they satisfy

$$
\left\{\begin{array}{l}
\alpha^{\prime \prime}(t)+K \alpha(t) \geq f\left(t, \alpha(t), \alpha^{\prime}(t), \beta(t)\right)+K \beta(t), \quad t \in(a, b), \\
\alpha(a)=\alpha(b), \quad \alpha^{\prime}(a) \geq \alpha^{\prime}(b), \\
\beta^{\prime \prime}(t)+K \beta(t) \leq f\left(t, \beta(t), \beta^{\prime}(t), \alpha(t)\right)+K \alpha(t), \quad t \in(a, b), \\
\beta(a)=\beta(b), \quad \beta^{\prime}(a) \leq \beta^{\prime}(b) .
\end{array}\right.
$$


For given $\alpha, \beta \in C[a, b]$, we shall write $\beta \leq \alpha$ if $\beta(t) \leq \alpha(t)$ for all $t \in[a, b]$. In such a case, we shall denote

$$
[\beta, \alpha]=\{u \in C[a, b]: \beta(t) \leq u(t) \leq \alpha(t), t \in[a, b]\}
$$

Assume that there exist constants $N>0, M, L \geq 0$ and $K>0$ such that $L-K \geq 0$, we need the following hypotheses:

(H1) For given $\alpha, \beta \in C[a, b]$ with $\beta \leq \alpha$,

$$
\left|f\left(t, x, y_{1}, z\right)-f\left(t, x, y_{2}, z\right)\right| \leq N\left|y_{1}-y_{2}\right|, \quad \forall x, z \in[\beta, \alpha], y_{1}, y_{2} \in \mathbb{R}
$$

(H2) For $\beta \leq x_{2} \leq x_{1} \leq \alpha, \beta \leq z_{1} \leq z_{2} \leq \alpha$,

$$
f\left(t, x_{1}, y, z_{1}\right)-f\left(t, x_{2}, y, z_{2}\right) \geq M\left(x_{1}-x_{2}\right)-L\left(z_{1}-z_{2}\right)
$$

(H3) $1-\frac{b-a}{\pi} N-\left(\frac{b-a}{\pi}\right)^{2} K>0$;

(H4) $b-a \leq 2 \theta\left(K, \frac{N}{2}\right)$, where $\theta$ is defined in (2.2).

In order to develop the monotone iterative technique for (1.3), we shall first consider the existence of solutions for the following periodic problems:

$$
\left\{\begin{array}{l}
u^{\prime \prime}(t)+K u(t)=F_{\xi, \eta}\left(t, u^{\prime}(t)\right), \quad t \in(a, b), \\
u(a)=u(b), \quad u^{\prime}(a)=u^{\prime}(b)
\end{array}\right.
$$

and

$$
\left\{\begin{array}{l}
v^{\prime \prime}(t)+K v(t)=F_{\eta, \xi}\left(t, v^{\prime}(t)\right), \quad t \in(a, b), \\
v(a)=v(b), \quad v^{\prime}(a)=v^{\prime}(b)
\end{array}\right.
$$

for each fixed $\xi, \eta \in[\beta, \alpha]$, here

$$
F_{\xi, \eta}(t, w)=f(t, \xi, w, \eta)+K \eta, \quad F_{\eta, \xi}(t, w)=f(t, \eta, w, \xi)+K \xi .
$$

Lemma 3.1 Assume that (H1), (H3) and (H4) hold. Then the problems (3.2) and (3.3) have unique solutions.

Proof In order to apply Lemma 2.1 to (3.2), we choose the Banach spaces $X=C^{1}[a, b]$ with the norm $\|u\|=\max \left\{\|u\|_{\infty},\left\|u^{\prime}\right\|_{\infty}\right\}$, where $\|u\|_{\infty}=\max _{t \in[a, b]}|u(t)|$, and $Y=C[a, b]$. Define the linear operator

$$
L: \operatorname{dom} L \subset X \rightarrow Y, \quad(L u)(t)=u^{\prime \prime}(t)
$$

and the nonlinear operator

$$
N: X \rightarrow Y, \quad(N u)(t)=F_{\xi, \eta}\left(t, u^{\prime}(t)\right)-K u(t),
$$


here,

$$
\operatorname{dom} L=\left\{u \in X: u \in C^{2}[a, b], u(a)=u(b), u^{\prime}(a)=u^{\prime}(b)\right\} .
$$

Let

$$
(P u)(t)=\bar{u}, \quad(Q u)(t)=\bar{u},
$$

where $\bar{u}=\frac{1}{b-a} \int_{a}^{b} u(s) d s$. Then, $\operatorname{ker} L=\{c \in \operatorname{dom} L: c \in \mathbb{R}\}$ and

$$
\operatorname{Im} L=\left\{u \in Y: \int_{a}^{b} u(s) d s=0\right\}
$$

Now, $\operatorname{Im} L$ is closed in $Y$. It is easy to see that $\operatorname{Im} L=\operatorname{ker} Q$ and $\operatorname{dim} \operatorname{ker} L=1=\operatorname{dim} \operatorname{Im} Q=$ codim $\operatorname{Im} L$. Thus, $L$ is a linear Fredholm operator with index zero.

Furthermore, let $L_{p}=\left.L\right|_{\operatorname{dom} L \cap \operatorname{ker} P}$ and $K_{p}: \operatorname{Im} L \rightarrow \operatorname{dom} L \cap \operatorname{ker} P$ denoting the inverse of $L_{p}$ be given by

$$
\left(K_{p} u\right)(t)=\int_{a}^{b} k(t, s) u(s) d s
$$

where

$$
k(t, s):=\frac{1}{2(b-a)} \begin{cases}(s-a)(2 t-b-s), & a \leq s<t \leq b, \\ (b-s)(a+s-2 t), & a \leq t \leq s \leq b .\end{cases}
$$

Obviously, $Q N$ and $K_{p}(I-Q) N$ are continuous. By Arzelà-Ascoli theorem, we can show that $K_{p}(I-Q) N(\bar{\Omega})$ is relative compact for any open bounded set $\Omega \subset X$. Moreover, $Q N(\bar{\Omega})$ is bounded. Thus, $N$ is $L$-compact on $\bar{\Omega}$.

In the following, we complete the remainder proof by four steps.

Step 1. Define $\Omega_{1}=\{u \in \operatorname{dom} L \backslash \operatorname{ker} L: L u=\lambda N u, \lambda \in(0,1)\}$. For any $u \in \Omega_{1}$,

$$
u^{\prime \prime}(t)=\lambda F_{\xi, \eta}\left(t, u^{\prime}(t)\right)-\lambda K u(t)
$$

Integrating (3.4) on $[a, b]$ to obtain

$$
K \bar{u}=\frac{1}{b-a} \int_{a}^{b} F_{\xi, \eta}\left(s, u^{\prime}(s)\right) d s
$$

Set $x=u-\bar{u}$, then $x \in \operatorname{dom} L, \bar{x}=0, x^{\prime}=u^{\prime}$. It follows from (3.4) and (3.5) that

$$
x^{\prime \prime}(t)=\lambda F_{\xi, \eta}\left(t, x^{\prime}(t)\right)-\lambda K x(t)-\frac{\lambda}{b-a} \int_{a}^{b} F_{\xi, \eta}\left(s, x^{\prime}(s)\right) d s .
$$

Notice that $\bar{x}=0$, by Lemma 2.3 , we have

$$
\|x\|_{2} \leq \frac{b-a}{\pi}\left\|x^{\prime}\right\|_{2}, \quad\|x\|_{\infty} \leq \sqrt{b-a}\left\|x^{\prime}\right\|_{2} .
$$


In view of $(\mathrm{H} 1)$, we have

$$
\begin{aligned}
\left|F_{\xi, \eta}(t, w)\right| & =|f(t, \xi, w, \eta)+K \eta| \\
& \leq|f(t, \xi, w, \eta)-f(t, \xi, 0, \eta)|+|f(t, \xi, 0, \eta)|+K|\eta| \\
& \leq N|w|+C
\end{aligned}
$$

for some constant $C$. Multiplying (3.6) by $x(t)$ and integrating it on $[a, b]$, we can obtain

$$
\begin{aligned}
\int_{a}^{b}\left|x^{\prime}(t)\right|^{2} d t= & -\lambda \int_{a}^{b} x(t) F_{\xi, \eta}\left(t, x^{\prime}(t)\right) d t+\lambda K \int_{a}^{b}|x(t)|^{2} d t \\
\leq & K \int_{a}^{b}|x(t)|^{2} d t+N \int_{a}^{b}|x(t)|\left|x^{\prime}(t)\right| d t+C \int_{a}^{b}|x(t)| d t \\
\leq & K\left(\frac{b-a}{\pi}\right)^{2} \int_{a}^{b}\left|x^{\prime}(t)\right|^{2} d t+N\left(\int_{a}^{b}|x(t)|^{2} d t\right)^{\frac{1}{2}}\left(\int_{a}^{b}\left|x^{\prime}(t)\right|^{2} d t\right)^{\frac{1}{2}} \\
& +C \sqrt{b-a}\left(\int_{a}^{b}|x(t)|^{2} d t\right)^{\frac{1}{2}} \\
\leq & \left(K\left(\frac{b-a}{\pi}\right)^{2}+N \frac{b-a}{\pi}\right)\left\|x^{\prime}\right\|_{2}^{2}+C \frac{(b-a)^{\frac{3}{2}}}{\pi}\left\|x^{\prime}\right\|_{2} .
\end{aligned}
$$

Notice the condition (H3), we can find a constant $M_{1}>0$ such that $\left\|x^{\prime}\right\|_{2} \leq M_{1}$ and also $\|x\|_{\infty} \leq \sqrt{b-a} M_{1}$. Now,

$$
|\bar{u}| \leq \frac{1}{K(b-a)} \int_{a}^{b}\left|F_{\xi, \eta}\left(s, u^{\prime}(s)\right)\right| d s \leq \frac{N}{K(b-a)} \int_{a}^{b}\left|u^{\prime}(t)\right| d t+\frac{C}{K} \leq \frac{N M_{1}}{K \sqrt{b-a}}+\frac{C}{K}
$$

implies

$$
\|u\|_{\infty} \leq\|x\|_{\infty}+|\bar{u}| \leq \sqrt{b-a} M_{1}+\frac{N M_{1}}{K \sqrt{b-a}}+\frac{C}{K}=: M_{2} .
$$

Since $u(a)=u(b)$, there exists $t_{0} \in(a, b)$ such that $u^{\prime}\left(t_{0}\right)=0$. Then

$$
\begin{aligned}
\left|u^{\prime}(t)\right| & \leq \int_{a}^{b}\left|u^{\prime \prime}(t)\right| d t \\
& \leq \int_{a}^{b}\left|F_{\xi, \eta}\left(t, u^{\prime}(t)\right)\right| d t+K \int_{a}^{b}|u(t)| d t \\
& \leq N \sqrt{b-a} M_{1}+C(b-a)+K(b-a) M_{2} \\
& =: M_{3},
\end{aligned}
$$

i.e. $\left\|u^{\prime}\right\|_{\infty} \leq M_{3}$. Hence, $\Omega_{1}$ is bounded.

Step 2. Let $\Omega_{2}=\{u \in \operatorname{ker} L: Q N x=0\}$. For $u \in \Omega_{2}$, we have $u=c \in \mathbb{R}$, and

$$
Q N c=\frac{1}{b-a} \int_{a}^{b}\left[F_{\xi, \eta}(t, 0)-K c\right] d t=0 .
$$


Then

$$
c=\frac{1}{K(b-a)} \int_{a}^{b} F_{\xi, \eta}(t, 0) d t \quad \text { and so } \quad|c| \leq \frac{C}{K} \leq M_{2}
$$

that is, $\Omega_{2}$ is bounded.

Step 3. Let $\Omega \supset \Omega_{1} \cup \Omega_{2}$ with $\Omega$ open and bounded. Clearly, conditions (A1) and (A2) in Lemma 2.1 are satisfied. The remainder is to verify (A3). To this end, we define an isomorphism $J: \operatorname{Im} Q \rightarrow \operatorname{ker} L$ by $J=I$. Let

$$
H(u, \mu)=\mu u+(1-\mu) J Q N u, \quad(u, \mu) \in \bar{\Omega} \times[0,1],
$$

i.e.

$$
H(u, \mu)=\mu u+\frac{1-\mu}{b-a} \int_{a}^{b}\left(F_{\xi, \eta}\left(s, u^{\prime}(s)\right)-K u(s)\right) d s .
$$

It is easy to see that $H(u, \mu) \neq 0$ for $(u, \mu) \in(\partial \Omega \cap \operatorname{ker} L) \times[0,1]$. Hence,

$$
\begin{aligned}
& \operatorname{deg}\left\{\left.J Q N\right|_{\bar{\Omega} \cap \operatorname{ker} L}, \Omega \cap \operatorname{ker} L, 0\right\} \\
& =\operatorname{deg}\{H(\cdot, 0), \Omega \cap \operatorname{ker} L, 0\} \\
& =\operatorname{deg}\{H(\cdot, 1), \Omega \cap \operatorname{ker} L, 0\} \\
& =\operatorname{deg}\{I, \Omega \cap \operatorname{ker} L, 0\} \\
& \quad \neq 0 .
\end{aligned}
$$

Lemma 2.1 yields that $L u=N u$ has at least one solution.

Step 4. We claim that the problem (3.2) has a unique solution. Suppose to the contrary, that there exist two solutions $u_{1}$ and $u_{2}$ of (3.2).

Let $u=u_{2}-u_{1}$, by (H1) and (H4),

$$
\begin{aligned}
u^{\prime \prime}(t)+K u(t) & =F_{\xi, \eta}\left(t, u_{2}^{\prime}(t)\right)-F_{\xi, \eta}\left(t, u_{1}^{\prime}(t)\right) \\
& =f\left(t, \xi(t), u_{2}^{\prime}(t), \eta(t)\right)-f\left(t, \xi(t), u_{1}^{\prime}(t), \eta(t)\right) \\
& \geq-N\left|u_{2}^{\prime}(t)-u_{1}^{\prime}(t)\right| \\
& =-N\left|u^{\prime}(t)\right| .
\end{aligned}
$$

Applying Lemma 2.2, we have $u(t) \geq 0$ on $[a, b]$. That is, $u_{2} \geq u_{1}$. Also, we can obtain $u_{1} \geq u_{2}$ by the same method. Thus, $u_{1} \equiv u_{2}$.

By a similar argument, we can obtain the existence of unique solution for the problem (3.3). 
Now, we investigate the extremal solution of the periodic system (1.3). For given $\alpha, \beta \in X$, we consider the approximation schemes

$$
\left\{\begin{array}{l}
\alpha_{0}=\alpha, \quad \beta_{0}=\beta, \\
\alpha_{n}^{\prime \prime}+K \alpha_{n}=f\left(t, \alpha_{n-1}, \alpha_{n}^{\prime}, \beta_{n-1}\right)+K \beta_{n-1}, \\
\beta_{n}^{\prime \prime}+K \beta_{n}=f\left(t, \beta_{n-1}, \beta_{n}^{\prime}, \alpha_{n-1}\right)+K \alpha_{n-1}, \\
\alpha_{n}(a)=\alpha_{n}(b), \quad \alpha_{n}^{\prime}(a)=\alpha_{n}^{\prime}(b), \\
\beta_{n}(a)=\beta_{n}(b), \quad \beta_{n}^{\prime}(a)=\beta_{n}^{\prime}(b),
\end{array}\right.
$$

Now, we give the main result for (1.3).

Theorem 3.1 Assume that $\alpha, \beta$ are a pair of lower and upper solutions of (1.3) defined by (3.1) such that $\beta \leq \alpha$. Suppose that (H1)-(H4) hold.

Then there exist two monotone iterative sequences $\left\{\alpha_{n}\right\},\left\{\beta_{n}\right\} \subset[\beta, \alpha]$, non-increasing and nondecreasing, respectively, defined by (3.7) such that $\alpha_{n} \rightarrow u^{*}, \beta_{n} \rightarrow v^{*}(n \rightarrow \infty)$ uniformly on $t \in[a, b]$, and the pair $\left(u^{*}, v^{*}\right)$ is a solution of $(1.3)$ satisfying

$$
\beta \leq v^{*} \leq u^{*} \leq \alpha .
$$

Moreover, any solution $(u, v)$ of $(1.3)$ with $\beta \leq u \leq \alpha, \beta \leq v \leq \alpha$ is such that

$$
v^{*} \leq u \leq u^{*}, \quad v^{*} \leq v \leq u^{*}
$$

Proof Let $R=\{(u, v) \in C[a, b] \times C[a, b]: u \geq 0, v \leq 0\}$ be an ordered normal cone, the order is defined by

$$
\left(u_{1}, v_{1}\right) \leq\left(u_{2}, v_{2}\right) \quad \Longleftrightarrow \quad u_{1} \leq u_{2}, \quad v_{1} \geq v_{2}
$$

for any $\left(u_{1}, v_{1}\right),\left(u_{2}, v_{2}\right) \in R$. We define the operator $T:[\beta, \alpha] \times[\beta, \alpha] \rightarrow X \times X$ by $T(\xi, \eta)=$ $(u, v)$, where $u$ and $v$ are the unique solutions of problems (3.2) and (3.3), respectively, with given $\xi, \eta \in[\beta, \alpha]$. Firstly, we claim that the mapping $T$ has the following properties:

(i) $(\beta, \alpha) \leq T(\alpha, \beta) \leq(\alpha, \beta)$;

(ii) $T\left(\xi_{1}, \eta_{1}\right) \leq T\left(\xi_{2}, \eta_{2}\right)$, when $\left(\xi_{1}, \eta_{1}\right) \leq\left(\xi_{2}, \eta_{2}\right), \xi_{i}, \eta_{i} \in[\beta, \alpha], i=1,2$.

We start with (i). Set $\left(\alpha_{1}, \beta_{1}\right)=T(\alpha, \beta), w=\alpha-\alpha_{1}$. From (H1) and (H2), we have

$$
\begin{aligned}
w^{\prime \prime}(t)+K w(t) & \geq f\left(t, \alpha(t), \alpha^{\prime}(t), \beta(t)\right)+K \beta(t)-f\left(t, \alpha(t), \alpha_{1}^{\prime}(t), \beta(t)\right)-K \beta(t) \\
& \geq-N\left|\alpha^{\prime}(t)-\alpha_{1}^{\prime}(t)\right|=-N\left|w^{\prime}(t)\right|, \quad t \in(a, b) .
\end{aligned}
$$

Obviously, $w(a)=w(b)$ and $w^{\prime}(a) \geq w^{\prime}(b)$. By Lemma 2.2, we have $w \geq 0$, and so $\alpha \geq \alpha_{1}$. A similar argument shows that $\beta_{1} \geq \beta$. Thus, $T(\alpha, \beta) \leq(\alpha, \beta)$. Set $w=\alpha_{1}-\beta$, then

$$
\begin{aligned}
w^{\prime \prime}(t)+K w(t) \geq & f\left(t, \alpha(t), \alpha_{1}^{\prime}(t), \beta(t)\right)+K \beta(t)-f\left(t, \beta(t), \beta^{\prime}(t), \alpha(t)\right)-K \alpha(t) \\
= & {\left[f\left(t, \alpha(t), \alpha_{1}^{\prime}(t), \beta(t)\right)-f\left(t, \alpha(t), \beta^{\prime}(t), \beta(t)\right)\right] } \\
& +\left[f\left(t, \alpha(t), \beta^{\prime}(t), \beta(t)\right)-f\left(t, \beta(t), \beta^{\prime}(t), \alpha(t)\right)\right]-K(\alpha(t)-\beta(t))
\end{aligned}
$$




$$
\begin{aligned}
& \geq-N\left|\alpha_{1}^{\prime}(t)-\beta(t)\right|+(M+L-K)(\alpha(t)-\beta(t)) \\
& \geq-N\left|w^{\prime}(t)\right|, \quad t \in(a, b) .
\end{aligned}
$$

Lemma 2.2 implies that $\alpha_{1} \geq \beta$. Similarly, we can check that $\alpha \geq \beta_{1}$. Hence, $(\beta, \alpha) \leq$ $T(\alpha, \beta)$.

Now, we prove (ii). Let $T\left(\xi_{i}, \eta_{i}\right)=\left(u_{i}, v_{i}\right), i=1,2$. Set $w=u_{2}-u_{1}$,

$$
\begin{aligned}
w^{\prime \prime}(t)+K w(t)= & F_{\xi_{2}, \eta_{2}}\left(t, u_{2}^{\prime}(t)\right)-F_{\xi_{1}, \eta_{1}}\left(t, u_{1}^{\prime}(t)\right) \\
= & f\left(t, \xi_{2}(t), u_{2}^{\prime}(t), \eta_{2}(t)\right)-f\left(t, \xi_{1}(t), u_{1}^{\prime}(t), \eta_{1}(t)\right)-K\left(\eta_{1}(t)-\eta_{2}(t)\right) \\
= & {\left[f\left(t, \xi_{2}(t), u_{2}^{\prime}(t), \eta_{2}(t)\right)-f\left(t, \xi_{2}(t), u_{1}^{\prime}(t), \eta_{2}(t)\right)\right] } \\
& +\left[f\left(t, \xi_{2}(t), u_{1}^{\prime}(t), \eta_{2}(t)\right)-f\left(t, \xi_{1}(t), u_{1}^{\prime}(t), \eta_{1}(t)\right)\right]-K\left(\eta_{1}(t)-\eta_{2}(t)\right) \\
\geq & -N\left|u_{2}^{\prime}(t)-u_{1}^{\prime}(t)\right|+M\left(\xi_{2}(t)-\xi_{1}(t)\right)+(L-K)\left(\eta_{1}(t)-\eta_{2}(t)\right) \\
\geq & -N\left|w^{\prime}(t)\right|, \quad t \in(a, b) .
\end{aligned}
$$

Applying Lemma 2.2, we get $u_{2} \geq u_{1}$. A similar argument shows that $v_{1} \geq v_{2}$. Thus, $T\left(\xi_{2}, \eta_{2}\right) \geq T\left(\xi_{1}, \eta_{1}\right)$.

To prove the sequence $\left\{\alpha_{n}\right\}$ and $\left\{\beta_{n}\right\}$ are bounded, we define $\left\{\left(\alpha_{n}, \beta_{n}\right)\right\}$ by $\left(\alpha_{n+1}, \beta_{n+1}\right)=$ $T\left(\alpha_{n}, \beta_{n}\right)$. Notice that $\left\{\left(\alpha_{n}, \beta_{n}\right)\right\}$ is non-increasing and $\left\{\left(\beta_{n}, \alpha_{n}\right)\right\}$ is non-decreasing. It is clear that $\left\{\alpha_{n}\right\}$ and $\left\{\beta_{n}\right\}$ are bounded. Hence, there exists a constant $C_{1}>0$ such that $\max \left\{\left\|\alpha_{n}\right\|_{\infty},\left\|\beta_{n}\right\|_{\infty}\right\} \leq C_{1}$ for all $n \in \mathbb{N}$, and

$$
\begin{aligned}
& \alpha_{n+1}^{\prime \prime}(t)+K \alpha_{n+1}(t)=F_{\alpha_{n}, \beta_{n}}\left(t, \alpha_{n+1}^{\prime}(t)\right), \\
& \beta_{n+1}^{\prime \prime}(t)+K \beta_{n+1}(t)=F_{\beta_{n}, \alpha_{n}}\left(t, \beta_{n+1}^{\prime}(t)\right) .
\end{aligned}
$$

Essentially, the same as in the proof of Lemma 3.1 guarantees that there exists a constant $C_{2}>0$ such that $\max \left\{\left\|\alpha_{n}^{\prime}\right\|_{\infty},\left\|\beta_{n}^{\prime}\right\|_{\infty}\right\} \leq C_{2}$. This shows that $\left\{\left(\alpha_{n}, \beta_{n}\right)\right\}$ converges in $X$, i.e. there exists $\left(u^{*}, v^{*}\right) \in X$ so that $\left(\alpha_{n}, \beta_{n}\right) \rightarrow\left(u^{*}, v^{*}\right), \beta_{0} \leq u^{*} \leq \alpha_{0}, \beta_{0} \leq v^{*} \leq \alpha_{0}$, and also $\beta_{0} \leq v^{*} \leq u^{*} \leq \alpha_{0}$ from (i).

Finally, we will prove that any other solution $(u, v) \in X$ of $(1.3)$ such that $\left(\beta_{0}, \alpha_{0}\right) \leq(u, v) \leq$ $\left(\alpha_{0}, \beta_{0}\right)$ satisfies

$$
v^{*} \leq v \leq u^{*}, \quad v^{*} \leq u \leq u^{*} .
$$

We can proceed as in the proof of (i) to show that $\left(\beta_{n}, \alpha_{n}\right) \leq(u, v) \leq\left(\alpha_{n}, \beta_{n}\right)$, i.e. $\beta_{n} \leq$ $u \leq \alpha_{n}$ and $\beta_{n} \leq v \leq \alpha_{n}, n=0,1,2, \ldots$. Hence, $v^{*} \leq u \leq u^{*}$ and $v^{*} \leq v \leq u^{*}$, i.e. $\left(v^{*}, u^{*}\right) \leq$ $(u, v) \leq\left(u^{*}, v^{*}\right)$.

Remark 3.1 Comparing with the main result (Theorem 3.2) in [8], we construct the explicit expression of the monotone iterative sequences $\left\{\alpha_{n}\right\}$ and $\left\{\beta_{n}\right\}$ by (3.7) in Theorem 3.1, while reference [8] didn't do so.

Remark 3.2 Notice that if $(u, u)$ is a solution of the auxiliary system (1.3), then $u$ is a solution of the given problem (1.1) under the assumption $f(t, u, w, u) \equiv g(t, u, w)$. Thus, $u^{*}$ and $v^{*}$ are bounds on solutions of (1.1). 
If the bounds $u^{*}$ and $v^{*}$ obtained in Theorem 3.1 are equal, then $u^{*}=v^{*}$ is a unique solution of the problem (1.1). In particular, under appropriate assumptions, this theorem provides an approximation scheme to the unique solution of (1.1).

\section{Unique solution for (1.1)}

Theorem 4.1 Suppose that the conditions (H1)-(H4) hold. Then the periodic problem (1.1) has a unique solution provided that $M+L>2 K+\frac{N^{2}}{4}$.

Proof If $u^{*}>v^{*}$, we compute

$$
\begin{aligned}
& \int_{a}^{b}\left[\left(\left(v^{*}(t)\right)^{\prime \prime}-\left(u^{*}(t)\right)^{\prime \prime}\right)+K\left(v^{*}(t)-u^{*}(t)\right)\right]\left(u^{*}(t)-v^{*}(t)\right) d t \\
& =\int_{a}^{b}\left[\left(f\left(t, v^{*}(t),\left(v^{*}(t)\right)^{\prime}, u^{*}(t)\right)-f\left(t, u^{*}(t),\left(u^{*}(t)\right)^{\prime}, v^{*}(t)\right)\right)\right. \\
& \left.\quad+K\left(u^{*}(t)-v^{*}(t)\right)\right]\left(u^{*}(t)-v^{*}(t)\right) d t \\
& \leq-(M+L-K) \int_{a}^{b}\left|u^{*}(t)-v^{*}(t)\right|^{2} d t \\
& \quad+N\left(\int_{a}^{b}\left|u^{*}(t)-v^{*}(t)\right|^{2} d t\right)^{\frac{1}{2}}\left(\int_{a}^{b}\left|\left(v^{*}(t)\right)^{\prime}-\left(u^{*}(t)\right)^{\prime}\right|^{2} d t\right)^{\frac{1}{2}} \\
& =:
\end{aligned}
$$

On the other hand,

$$
\begin{aligned}
& -\int_{a}^{b}\left[\left(\left(v^{*}(t)\right)^{\prime \prime}-\left(u^{*}(t)\right)^{\prime \prime}\right)+K\left(v^{*}(t)-u^{*}(t)\right)\right]\left(v^{*}(t)-u^{*}(t)\right) d t \\
& \quad=\int_{a}^{b}\left|\left(v^{*}(t)\right)^{\prime}-\left(u^{*}(t)\right)^{\prime}\right|^{2} d t-K \int_{a}^{b}\left|v^{*}(t)-u^{*}(t)\right|^{2} d t \\
& \quad=: Q_{2} .
\end{aligned}
$$

If we denote $\gamma_{1}=\left(\int_{a}^{b}\left|u^{*}(t)-v^{*}(t)\right|^{2} d t\right)^{\frac{1}{2}}$ and $\gamma_{2}=\left(\int_{a}^{b}\left|\left(u^{*}(t)\right)^{\prime}-\left(v^{*}(t)\right)^{\prime}\right|^{2} d t\right)^{\frac{1}{2}}$, then

$$
\begin{aligned}
Q_{2}-Q_{1} & =\gamma_{2}^{2}-K \gamma_{1}^{2}+(M+L-K) \gamma_{1}^{2}-N \gamma_{1} \gamma_{2} \\
& =\left(\gamma_{2}-\frac{N}{2} \gamma_{1}\right)^{2}+\left(M+L-2 K-\frac{N^{2}}{4}\right) \gamma_{1}^{2} \\
& >0
\end{aligned}
$$

which is a contradiction.

Example 4.1 Consider the problem

$$
\left\{\begin{array}{l}
u^{\prime \prime}(t)=g\left(t, u(t), u^{\prime}(t)\right), \quad t \in(0,2 \pi), \\
u(0)=u(2 \pi), \quad u^{\prime}(0)=u^{\prime}(2 \pi) .
\end{array}\right.
$$


Corresponding to the problem (1.1), we take $a=0, b=2 \pi$ and

$$
g(t, x, y)=\frac{t}{2 \pi \times 10^{2}}+\frac{2 \pi^{2}}{500} x-\frac{1}{4}\left(\frac{\pi^{2}}{400}\right)^{4} x^{4}+\frac{\pi}{10 \sqrt{2}} \arctan y .
$$

Let

$$
f(t, x, y, z)=\frac{t}{2 \pi \times 10^{2}}+\frac{2 \pi^{2}}{500} x-\frac{1}{4}\left(\frac{\pi^{2}}{400}\right)^{4} z^{4}+\frac{\pi}{10 \sqrt{2}} \arctan y .
$$

Consider the following system:

$$
\begin{cases}u^{\prime \prime}(t)+\frac{\pi^{2}}{400} u(t)=f\left(t, u(t), u^{\prime}(t), v(t)\right)+\frac{\pi^{2}}{400} v(t), & t \in(0,2 \pi), \\ v^{\prime \prime}(t)+\frac{\pi^{2}}{400} v(t)=f\left(t, v(t), v^{\prime}(t), u(t)\right)+\frac{\pi^{2}}{400} u(t), & t \in(0,2 \pi), \\ u(0)=u(2 \pi), \quad u^{\prime}(0)=u^{\prime}(2 \pi), & \\ v(0)=v(2 \pi), \quad v^{\prime}(0)=v^{\prime}(2 \pi) & \end{cases}
$$

with $K=\frac{\pi^{2}}{400}$ corresponding to (1.3). Clearly, the system (4.3) has $\alpha=\frac{400}{\pi^{2}}$ and $\beta=-\frac{400}{\pi^{2}}$ as the lower and upper solutions respectively according to the definition by (3.1). Take $M=\frac{2 \pi^{2}}{500}, N=\frac{\pi}{10 \sqrt{2}}$ and $L=K=\frac{\pi^{2}}{400}$. It is easy to check that the conditions (H1)-(H3) hold. Since $K>\frac{N^{2}}{4}$,

$$
\theta\left(K, \frac{N^{2}}{4}\right)=\frac{1}{\sqrt{K-\frac{N^{2}}{4}}}\left(\frac{\pi}{2}-\arctan \frac{\frac{N}{2}}{\sqrt{K-\frac{N^{2}}{4}}}\right)=5 \sqrt{2} \geq \pi .
$$

Thus, (H4) is satisfied. Thanks to Theorems 3.1, the system (4.3) has a solution $\left(u^{*}, v^{*}\right)$. In view of the values of $N, K, M, L$, we have $M+L>2 K+\frac{N^{2}}{4}$. Hence, Theorem 4.1 implies that $u^{*}=v^{*}$, that is, the problem (4.1) has a unique solution.

Remark 4.1 When $\alpha=\frac{400}{\pi^{2}}, \beta=-\frac{400}{\pi^{2}}$, and $g$ is given by (4.3), Example 4.1 does not satisfy the conditions imposed in [1, Theorem 3.3] because $\alpha, \beta$ are not a pair of lower and upper solutions of Eq. (3.7) in [1]. In fact, our definitions of the lower and upper $\alpha, \beta$, and the monotone iterative sequences $\left\{\alpha_{n}\right\},\left\{\beta_{n}\right\}$ are all different from [1, Theorem 3.3]. In addition, the ultimate goal of this paper is the unique solution of (1.1) by constructing an auxiliary system (1.3), which is one of the highlights of this article.

\section{Competing interests}

The authors declare that they have no competing interests.

\section{Authors' contributions}

All authors contributed equally to the manuscript and read and approved the final manuscript.

\section{Acknowledgements}

The authors would like to express their sincere gratitude to the anonymous reviewers for their insightful comments and constructive suggestions, which helped to enrich the content and considerably improved the presentation of this paper. This research is supported by the National Natural Sciences Foundation of People's Republic of China (Grant No. 11226148 and No. 61273016), the Scientific Research Foundation of Zhejiang Province (Grant No. LY12F05006), and the Education Department Foundation of Zhejiang Province (Grant No. Y201121906). 


\section{References}

1. Coster, CD, Habets, P: Two-Point Boundary Value Problems: Lower and Upper Solutions. Mathematics in Science and Engineering, vol. 205. Elsevier, Amsterdam (2006)

2. Cabada, A, Habets, P, Pouso, RL: Optimal existence conditions for $p$-Laplacian equations with upper and lower solutions in the reverse order. J. Differ. Equ. 166, 385-401 (2000)

3. Cabada, A, Habets, P, Lois, S: Monotone method for the Neumann problem with lower and upper solutions in the reverse order. Appl. Math. Comput. 117, 1-14 (2001)

4. Torres, PJ, Zhang, M: A monotone iterative scheme for a nonlinear second order equation based on a generalized anti-maximum principle. Math. Nachr. 251, 101-107 (2003)

5. Jiang, D, Yang, Y, Chu, J, O'Regan, D: The monotone method for Neumann functional differential equations with upper and lower solutions in the reverse order. Nonlinear Anal. 67, 2815-2828 (2007)

6. Cabada, A, Grossinho, MR, Minhos, F: Extremal solutions for third-order nonlinear problems with upper and lower solutions in reversed order. Nonlinear Anal. 62, 1109-1121 (2005)

7. Li, F, Jia, M, Liu, X, Li, C, Li, G: Existence and uniqueness of solutions of second-order three-point boundary value problems with upper and lower solutions in the reversed order. Nonlinear Anal. 68, 2381-2388 (2008)

8. Zuo, WJ, Wang, PX, Jiang, DQ: Periodic boundary value problems and periodic solutions of second order FDE with upper and lower solutions. Southeast Asian Bull. Math. 30, 199-207 (2006)

9. Wang, W, Yang, X, Shen, J: Boundary value problems involving upper and lower solutions in reverse order. J. Comput. Appl. Math. 230, 1-7 (2009)

10. Khan, RA, Webb, JRL: Existence of at least three solutions of a second-order three-point boundary value problem. Nonlinear Anal. 64, 1356-1366 (2006)

11. Khan, RA, Webb, JRL: Existence of at least three solutions of nonlinear three point boundary value problems with super-quadratic growth. J. Math. Anal. Appl. 328, 690-698 (2007)

12. Khan, RA, Webb, JRL: Existence of at least two solutions of second order nonlinear three point boundary value problems. Dyn. Syst. Appl. 15, 119-132 (2006)

13. Yang, $A, G e, W$ : The monotone method for periodic differential equations with the non well-ordered upper and lower solutions. J. Comput. Appl. Math. 232, 632-637 (2009)

14. Mawhin, J: Topological Degree Methods in Nonlinear Boundary Value Problems. NSFCBMS Regional Conference Series in Mathematics. Am. Math. Soc., Providence (1979)

\section{Submit your manuscript to a SpringerOpen ${ }^{\circ}$ journal and benefit from:}

- Convenient online submission

- Rigorous peer review

- Immediate publication on acceptance

- Open access: articles freely available online

- High visibility within the field

- Retaining the copyright to your article 